\title{
Evaluación Comparativa de dos Alternativas de Infraestructura Tipo Túnel a partir de un Análisis de Accesibilidad Urbana
}

\author{
Sebastián Martínez, Diego A. Escobar y Jhonny A. Tamayo \\ Facultad de Ingeniería y Arquitectura, Universidad Nacional de Colombia, Sede Manizales.- Colombia \\ (e-mail: semartinezca@unal.edu.co, daescobarga@unal.edu.co, jatamayoar@unal.edu.co)
}

Recibido Sep. 7, 2016; Aceptado Nov. 15, 2016; Versión final Ene. 3, 2017, Publicado Ago. 2017

\begin{abstract}
Resumen
En esta investigación se evalúa el impacto que podría generar la inserción de una infraestructura tipo túnel en las condiciones de accesibilidad urbana que actualmente ofrece la red vial de la ciudad de Manizales en Colombia. Se analizan dos opciones que conectan el sector sur con el norte. El impacto se evalúa a partir del cálculo de los porcentajes de ahorro en los tiempos medios de viaje que se tendrían al construir una u otra alternativa y su relación con la cobertura poblacional según el estrato. Se encontró que una de las alternativas (B) representa unos mayores porcentajes de ahorro y beneficia en mayor porcentaje a los estratos medios. Sin embargo, se estima que el costo de su construcción es un $63 \%$ mayor al costo de construir la otra alternativa (A). El análisis de accesibilidad se realiza a partir de la aplicación de modelos Geoestadístico complementados con el uso de sistemas de información geográfica.
\end{abstract}

\section{Comparative Evaluation of Two Tunnel type Alternative Infrastructure from an Urban Accessibility Analysis}

\begin{abstract}
In this research the impact generated by the insertion of tunnel infrastructure related with the urban accessibility conditions currently offered by the road network of the city of Manizales, in Colombia, is evaluated. Two alternatives, A and B, for connecting the southern with the northern sectors of the city are evaluated. The impact is evaluated from the calculation of percentages of saving time that the people gain with the construction of alternatives $A$ and $B$, and its relationship with the population coverage by stratum. It was found that the second alternative B allows having higher percentages of saving time and benefits to a greater percentage of the medium social stratum people. However, it is estimated that the cost of its construction is $63 \%$ higher than the cost of built the alternative A. The accessibility analysis is done with the application of geostatistical models complemented with the use of geographic information systems.
\end{abstract}




\section{INTRODUCCIÓN}

La conurbación Manizales - Villamaría se encuentra localizada en la región centro occidente de Colombia sobre la cordillera central (2150 m.s.n.m, Ver Figura 1); en ésta habitan aproximadamente 415,000 personas en un área de $58.7 \mathrm{Km}^{2}$. El desarrollo urbano se adapta orgánicamente a una topografía abrupta, teniendo una morfología en forma de cometa en el sentido occidente-oriente, debiendo superar grandes dificultades topográficas en el sentido norte-sur, situación que ha incentivado en los últimos años, la implementación de sistemas de transporte tipo Cable aéreo (Escobar et al., 2015b). La actualidad de la ciudad en relación con la movilidad es que durante el año 2015 el sistema de transporte público colectivo de la ciudad registró una disminución del $5 \%$ en el número de pasajeros movilizados, el parque automotor registró un aumento de aproximadamente el $9 \%$, las motos se duplicaron en cinco años y los vehículos livianos aumentaron un $51 \%$ (MCV, 2016), para una red vial de aproximadamente 766 Km (Alcaldía de Manizales, 2011); a pesar que Manizales está identificada como una de las ciudades de Colombia en la cual existe una mayor proporción de personas que se transportan en modos de transporte no motorizados, la proporción de peatones para el año 2015 , disminuyó de $18 \%$ al $13 \%$, aunque se registró el aumento de un punto porcentual en el uso de la bicicleta ya que en dicho año fue puesto en marcha el Sistema Público de Bicicletas con 135 bicicletas ubicadas en ocho estaciones, que para marzo de 2016 ya contaba con más de dos mil viajes mensuales (MCV, 2016).
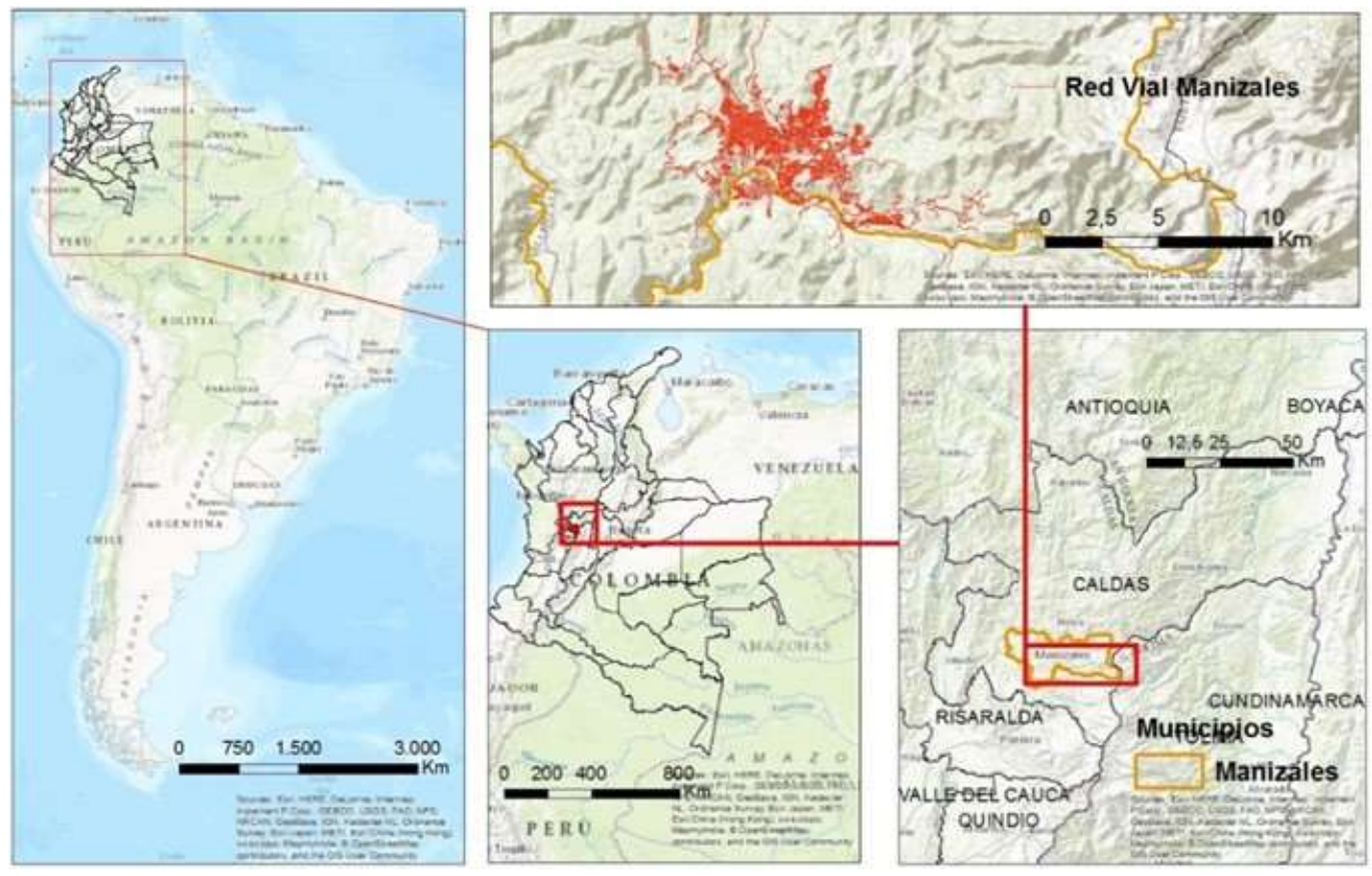

Fig. 1: Ubicación del área de estudio, conurbación Manizales - Villamaría

A lo largo de la historia del planeamiento regional y urbano, el termino de "accesibilidad" se ha utilizado en variados proyectos, se conocen aplicaciones de este término desde hace un siglo aproximadamente, periodo en el cual fue importante por su relación con teorías de localización y planeación económica regional (Batty, 2009). El concepto, de forma general, se entiende como una medida de la facilidad de comunicación existente entre una comunidad o actividad y un destino, comunicación que se realiza mediante el uso de diferentes modos de transporte (Morris et al., 1978); no obstante, la definición más clásica de este término, "...the potential of Opportunities for interaction. " (Hansen, 1959), se remonta a la década de los 60'. La accesibilidad ha sido un concepto aplicado en otros campos del conocimiento, como: desarrollo económico regional (Rietveld y Nijkamp, 1993; Vickerman et al., 1999; Mackinnion et al., 2008), sostenibilidad (Vega, 2011; Escobar et al., 2015a), demografía (Kotavaara, 2011), operatividad de modos de transporte (Botero et al., 2011; Escobar et al., 2012), cohesión social (López et al., 2008), localización de servicios (Park, 2012; Higgs, 2013; Younes et al., 2016), turismo (Kastenholz et al., 2012), entre otros campos.

Comúnmente, los estudios basados en medidas de accesibilidad se soportan en análisis de distancias y atracción de nodos que conforman una red (Curl et al., 2012), no obstante, existen parámetros que afectan la accesibilidad (Burkey, 2012) como la localización y número de nodos, la distribución poblacional, y la morfología y tipología de la red de transporte, parámetros que se complementan con los enmarcados en el nuevo paradigma de la movilidad, como son la proximidad de los usos del suelo y la calidad, costos, seguridad y comodidad de los modos de transporte, así como su influencia en el medioambiente (Litman, 2014). 
La accesibilidad territorial urbana debe ser reconocida como una necesidad primaria (Halden, 2011), pues por medio de ésta, es posible vislumbrar las variadas posibilidades que existen para alcanzar actividades específicas como salud, educación, empleo, dado un conjunto de individuos que se movilizan y necesitan acceder a dichos servicios o actividades. El conocer las condiciones de accesibilidad de un área se ha convertido en un elemento primordial de planificación y evaluación de proyectos de infraestructura (Gutiérrez et al., 2010), ya que a través de estos análisis es posible establecer criterios cuantitativos para la determinación del futuro uso de suelo, en miras a lograr un mayor bienestar social mediante una adecuada planificación sectorial (Kimbambe et al., 2013). Es así como los análisis de accesibilidad pueden soportar estudios relacionados con la inserción de infraestructuras (Zhu y Liu, 2004) y su impacto espacio - temporal sobre características sociodemográficas de una zona en particular (Chen et al., 2014; Karou y Hull, 2014).

Se ha comprobado que una forma de estimular el crecimiento económico de una región es generando inversión estratégica en infraestructura del transporte (Wong y Webb, 2014), para lo cual se debe tener en cuenta un desarrollo equitativo en cada una de las bases socioeconómicas con el fin de obtener modelos prácticos aplicables a la ciudad (Venegas y Rojas, 2009), teniéndose en cuenta las diferencias per cápita entre zonas, llamada estratificación social. En la Fig. 2 se observa la estratificación social actual de Manizales, los estratos 2 y 3 son los que mayor población albergan, como se observa en la Tabla 1 (la estratificación social va del estrato 1 al 6 según la capacidad económica del sector, siendo el estrato 1 la zona con capacidad baja y la zona estrato 6 de capacidad alta).

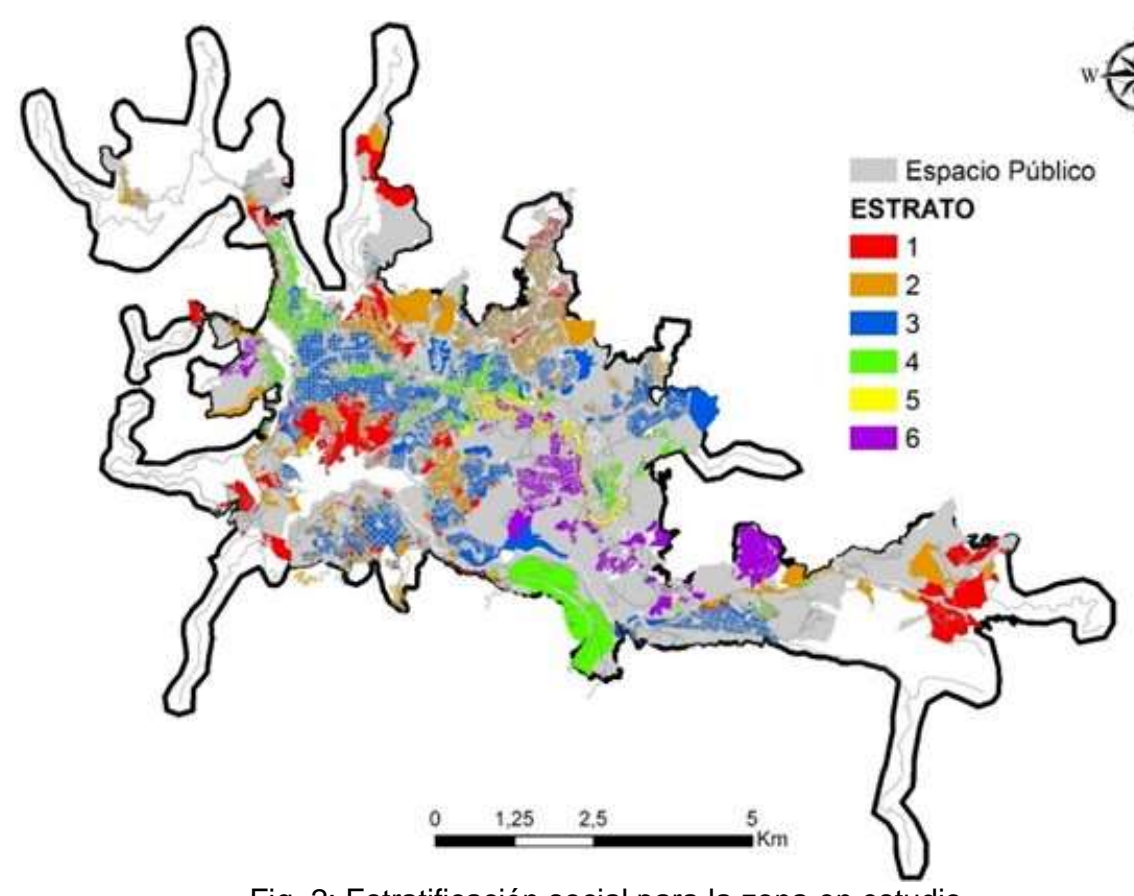

Fig. 2: Estratificación social para la zona en estudio

En la presente investigación se analizan dos propuestas de infraestructura vial tipo túnel, calculándose la reducción de tiempos medios de viaje y evaluando las condiciones de accesibilidad urbana que ofrecería la inserción de las obras, se comparan dos posibles alternativas que comunicaría dos corredores viales principales de la ciudad, proveyendo conexión de sur a norte y viceversa, buscando conocer los impactos que sobre cada uno de los estratos se tendría dada la construcción de una u otra alternativa de túnel. Lo anterior se relaciona directamente con la llamada justicia espacial, entendiendo ésta como el equilibrio en el acceso a cada una de las necesidades de igualdad económica y equipamientos en las diferentes zonas que conforman un territorio (Moreno y Angulo, 2009).

Tabla 1: Población oficial por cada estrato socioeconómico

\begin{tabular}{|c|c|c|c|c|c|c|}
\hline Hab./Estrato & Estrato 1 & Estrato 2 & Estrato 3 & Estrato 4 & Estrato 5 & Estrato 6 \\
\hline Población & 49,515 & 111,077 & 159,675 & 62,977 & 12,554 & 19,326 \\
\hline
\end{tabular}

Haciendo uso de los Sistemas de Información Geográfica (SIG) y mediante la aplicación de geoestadística, se calculan curvas isócronas que son base para el de cobertura espacial en relación directa con las variables sociodemográficas; los SIG permiten comprender fácilmente la información, para esta investigación en particular, apoyaron la ejecución de los algoritmos (Zhang et al., 2009) de caminos mínimos o trayectoria mínima desde cualquier nodo de la red a cualquier otro nodo de la red, los cuales proveen a los investigadores las herramientas necesarias para simular el impacto de inserción de una infraestructura. 
En Latinoamérica, el término accesibilidad ha sido poco abordado, desconociéndose el inmenso potencial que posee este tipo de metodologías, a pesar de ello, actualmente se poseen ejemplos reales de aplicación de análisis de accesibilidad a escala regional (Escobar et al., 2013) y a escala urbana (Escobar et al., 2016). En los siguientes capítulos se aborda la metodología aplicada, la formulación del modelo empleado, se discuten los principales resultados y se presentan las conclusiones.

\section{METODOLOGÍA}

En la Fig. 3 se presenta el diagrama de flujo que resume la metodología aplicada en la investigación, la cual se compone de cuatro etapas principales, a saber: 1) Actualización y validación de características operativas de la red de infraestructuras del transporte para los cálculos de Accesibilidad Territorial Urbana; 2) Cálculo de la Accesibilidad Media Global para el escenario actual de la ciudad de Manizales; 3) Cálculo de la Accesibilidad Media Global para los dos escenarios futuros propuestos; y 4) Análisis del gradiente para la Relación entre Accesibilidad Territorial Urbana del escenario Actual y los dos escenarios Futuros.

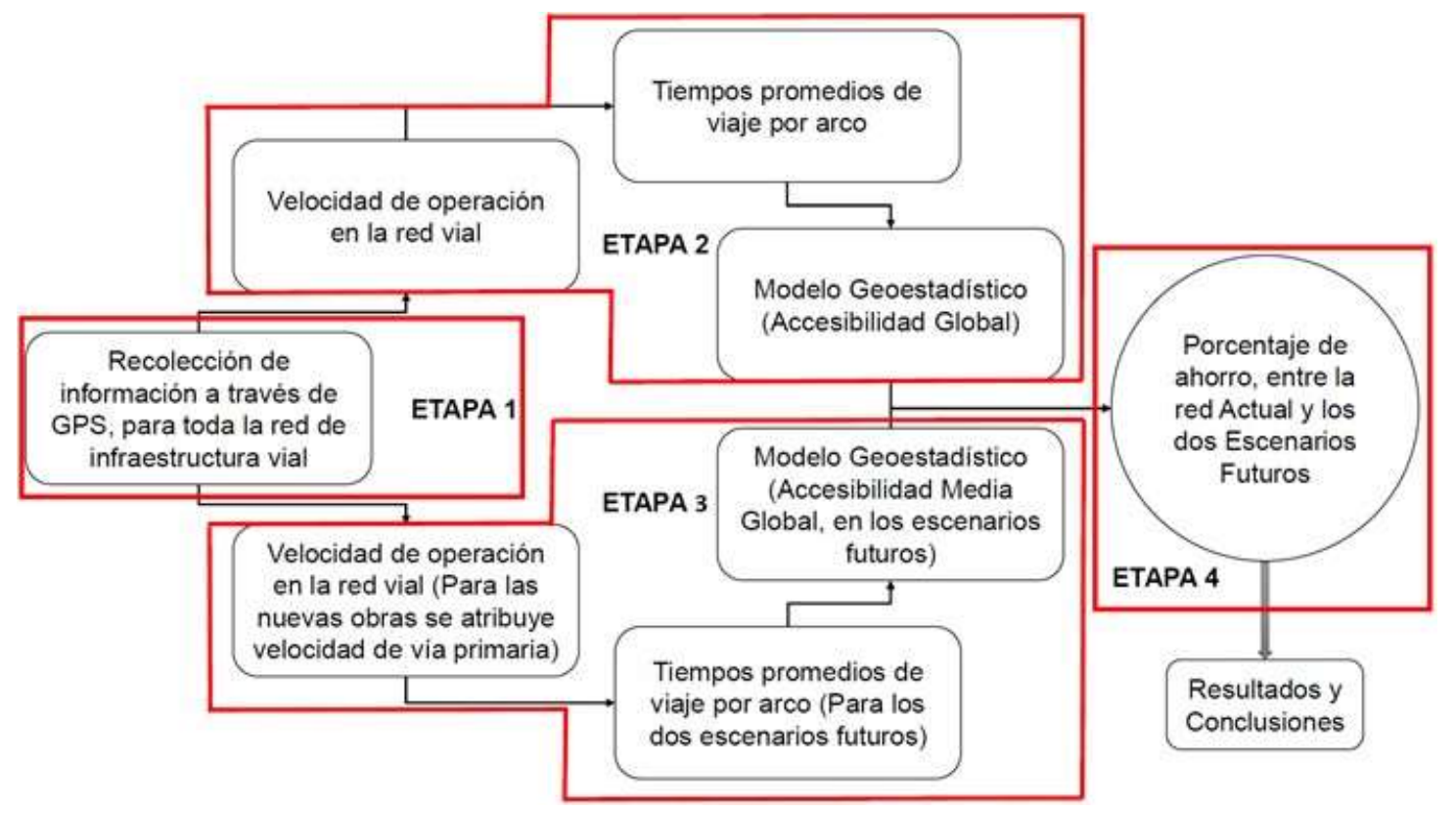

Fig. 3: Metodología aplicada

Etapa 1. Actualización y validación de características operativas de la red de infraestructuras del transporte para los cálculos de Accesibilidad Territorial Urbana: En esta etapa se tomó como información primaria la red de infraestructuras del transporte de la ciudad de Manizales (pública y privada), se usó un Sistema de Información Geográfica (SIG) que recogió la información de la red vial actual y la futura, según cada una de las alternativas a evaluarse; los SIG permiten comprender en un mayor detalle las características de accesibilidad ofrecidas por un modo de transporte, dada su capacidad de almacenamiento de información e integración de modelos relacionados con el territorio (Gómez y Barredor, 2005). El grafo ya validado se usó para el cálculo de la Accesibilidad territorial urbana a partir de datos de velocidad de operación, el cual está compuesto por más de 13,000 arcos y más de 8,750 nodos.

Etapa 2. Cálculo de la Accesibilidad Media Global para el escenario Actual de la ciudad de Manizales: Con la ayuda del software TransCAD® y la base de datos recolectada en la Etapa 1, se realiza el cálculo de los tiempos mínimos de viajes; luego, se estudia la interpolación en el software Surfer® para aplicar el método de Kriging Ordinario y analizar las curvas de tiempos mínimos de viajes, que comprenden desde cada nodo de la red hasta todos los nodos de la red; se analiza un mapa con información de área, población y vivienda, donde se detallan resultados concluyentes y por consiguiente, se analizan los tiempos mínimos de viajes de acuerdo a otra base de datos a partir de la estratificación de la ciudad.

Etapa 3. Cálculo de la Accesibilidad Media Global para los dos escenarios futuros propuestos: La tercera etapa es la relacionada con el cálculo de la Accesibilidad Territorial Urbana ofrecida por la red de infraestructuras de transporte actual en la ciudad de Manizales; teniendo en cuenta dos infraestructuras en la red vial, en primera instancia se propone un túnel desde parque centro en la vía panamericana (Sur) hasta las proximidades de la glorieta san Rafael (Norte oriente), con una longitud aproximada de $1.9 \mathrm{Km}$; como segunda alternativa estudiada se tiene un túnel desde parque centro en la vía panamericana (Sur) hasta las cercanías de la entrada al barrio Peralonso (Norte centro), con una longitud aproximada de 3.1 $\mathrm{Km}$. En ambos casos, la accesibilidad se analizó a partir del vector de tiempo medio de viaje (Tvi), 
obteniéndose las curvas isócronas que para los diferentes sectores de la ciudad se obtienen al usarse la red de infraestructuras del transporte propuesta para la ciudad.

Etapa 4. Análisis del gradiente para la Relación entre Accesibilidad Territorial Urbana del escenario Actual y los dos escenarios Futuros: En esta etapa, se relacionaron los resultados obtenidos en la Etapa 1 y en la etapa 2, según se analizan los tiempos mínimos de viaje para cada uno de los escenarios y así proporcionar porcentajes de ahorro, que serán consecuencia de las nuevas obras de infraestructura propuestas en la red de la ciudad de Manizales.

\section{FORMULACIÓN DEL MODELO}

Para el cálculo de la accesibilidad territorial urbana desde todos los nodos hacia todos los nodos de la red de infraestructura de transportes, se tienen los datos de velocidad de operación establecidos en el Plan de Movilidad vigente (Alcaldía de Manizales, 2011), lo cual permitió calcular los tiempos medios de viaje para cada segmento de red. El modelo que a continuación se formula, se basa en el propuesto por (Shen, 2002). De acuerdo a lo anterior, la Accesibilidad Territorial Urbana se analizó a partir del vector tiempo medio de viaje $\left(T v_{i}\right)$, el cual representa el tiempo promedio de viaje desde el nodo $i$ hasta los demás nodos de la red.

En el cálculo se utilizó un algoritmo del SIG que permite calcular la menor impedancia (Caminos Mínimos) entre un nodo específico y los demás nodos de la red, conformando una matriz unimodal de impedancias. A través de esta matriz y conociendo la velocidad de operación promedio de cada arco, se elaboró la matriz de tiempos promedios mínimos de viaje, en la que se minimiza el tiempo medio de viaje entre todos y cada uno de los nodos que conforman la red en estudio. Una vez determinada la matriz de tiempos promedios mínimos de viaje, se obtuvo el vector de tiempo promedio de viaje $T v_{i}\left(V e r\right.$ expresión (1)). Donde $T v_{i}=$ tiempo de viaje mínimo promedio entre el nodo $i$ y los demás nodos de la red $n=$ número de nodos de la red.

$\overline{T_{v l}}=\frac{\sum_{j=1}^{m} t_{v i}}{(n-1)}$, siendo $i=1,2,3, \ldots, n ; j=1,2,3, \ldots, m$.

El vector de Tiempos Mínimos de Viaje está representado por una matriz de tamaño [ $\left.n_{x} 1\right]$, la cual se relaciona con las coordenadas (longitud y latitud) de cada nodo de la red generándose una nueva matriz de tamaño [ $\left.n_{x} 3\right]$. El modelo geoestadístico Kriging ordinario con semivariograma lineal fue el escogido para la predicción de la variable TMV. Kriging Ordinario es un método geoestadístico utilizado para la estimación de valores desconocidos; con la creación de un semivariograma a partir de los puntos conocidos y en relación con el parámetro de ponderación de semivarianza media, se calculan los valores en los puntos desconocidos; la ecuación fundamental y básica del método donde $Z_{0}$ será el valor estimado para los puntos de interpolación es (Ver expresión (2)). $Z_{x}$ son los valores de los puntos conocidos, $W_{x}$ es la correlación 0 pesos de los puntos conocidos respecto a los puntos y $\mathrm{S}$ son los números de puntos conocidos.

$Z_{0}=\sum_{i=1}^{S} Z_{x} w_{x}$

Para la semivarianza media se tiene que $h$ es la distancia entre puntos, $\mathrm{n}$ son números pares de puntos que se definen según la distancia y z son los valores de puntos conocidos (Vorapracha et al., 2015) (Ver expresión (3)).

$f(h)=\frac{1}{2 n} \sum_{i=1}^{n}\left[z\left(x_{i}\right)-z\left(x_{i}+h\right)\right]^{2}$

La metodología aplicada toma las velocidades de operación promedio de los arcos que componen la red, lo cual se establece como un efecto simplificador en relación con la aplicación del modelo propuesto, ya que se está homogenizando el tiempo medio de viaje de todos los modos de transporte en un solo valor; esta situación debe tenerse en cuenta al momento de tomarse los resultados del presente modelo como base en procesos de planificación.

\section{PRINCIPALES RESULTADOS}

Accesibilidad Media Global ofrecida por la red vial actual. En la Fig. 4 se encuentra el resultado del modelo geoestadístico aplicado, donde se presentan las curvas isócronas de viaje entre todos los nodos de la red. Se destaca 70 min. como mayor tiempo medio de viaje obtenido, lo cual significa que desde dichos sitios, ubicados en la periferia de la ciudad en el sector occidente, es necesario invertir en promedio dicha cantidad de tiempo si se desea cubrir toda el área de estudio. Así mismo, se ubica en el plano las alternativas de túnel que serán evaluadas, alternativa 1 (línea roja) y alternativa 2 (línea azul). 
Se observa cómo la curva que representa los 25 min., se alarga más hacia el oriente que hacia el occidente, siguiendo dos corredores de movilidad principales, los cuales coinciden con el filo de la montaña sobre la cual reposa la ciudad. Al realizar los cálculos de cobertura espacial de las curvas isócronas en relación con las variables área, población y número de viviendas, se obtuvo que en el escenario actual, el $25 \%$ del área de estudio se cubre al invertirse unos $28 \mathrm{~min}$. de tiempo medio de viaje, mientras que para cubrir igual porcentaje de población y número de viviendas es necesario invertir $25 \mathrm{~min}$. de tiempo medio de viaje. En la Fig. 5 se observa la ojiva porcentual de distribución poblacional según estrato y curva isócrona que le cubre; se destaca el estrato 5 como el de mejor cobertura en el escenario actual, lo que significa que se cubre el mayor porcentaje de población de este estrato en menor cantidad de tiempo, esto, debido a las características de red de infraestructura vial que actualmente presenta la ciudad de Manizales. Así mismo, se observa que es el estrato 4 el que reporta condiciones de cobertura más desfavorables; encontrando, por ejemplo que para una cobertura del $50 \%$ de la población de cada estrato, el estrato 5 se cubriría con un tiempo medio de viaje de aproximadamente 24 min., los estratos 3, 6, 2 y 1 se cubrirían con tiempos medios de viaje de entre 27 y 28 min., mientras que el estrato 4 se cubriría con $31 \mathrm{~min}$. de tiempo medio de viaje.

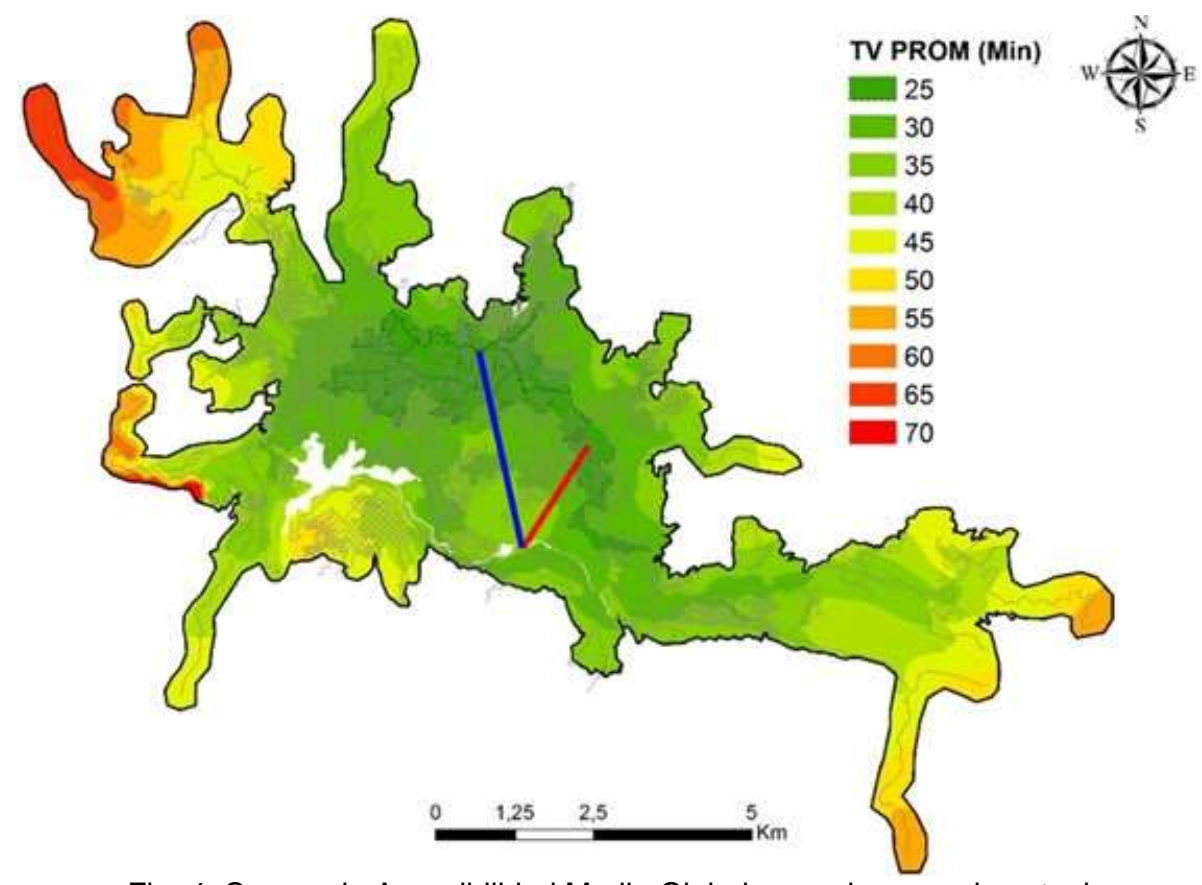

Fig. 4: Curvas de Accesibilidad Media Global para el escenario actual

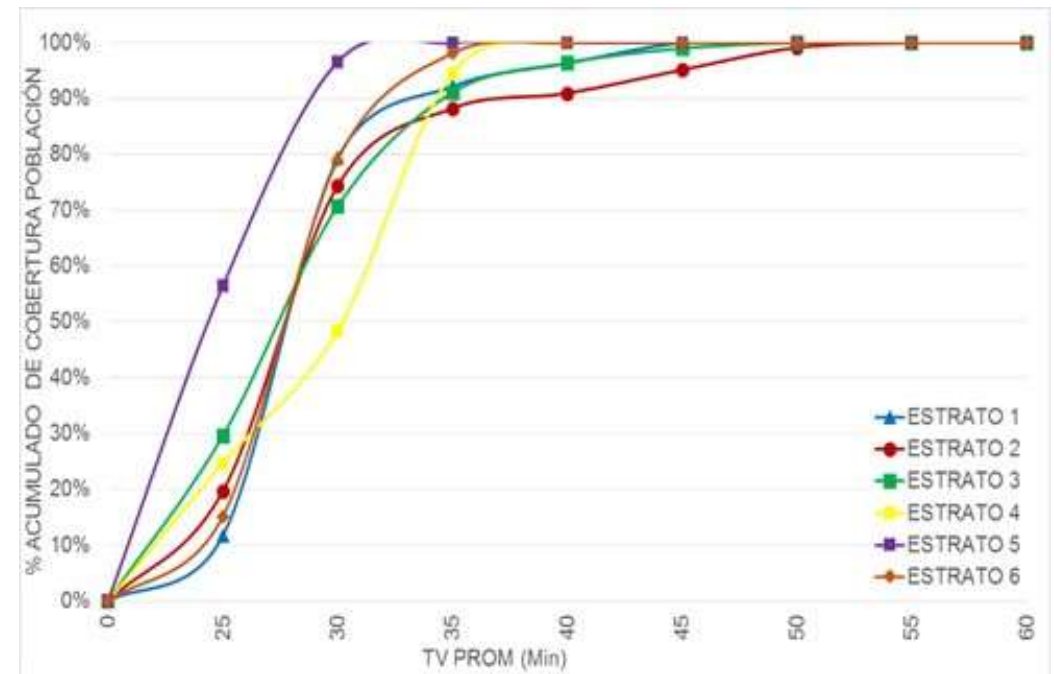

Fig. 5: Distribución porcentual de población por estratos para la zona de Estudio, escenario Actual

Accesibilidad Media Global ofrecida por la Alternativa 1 (Túnel Parque Centro - San Rafael) y evaluación del impacto en porcentaje de ahorro de tiempo. Para este escenario, la Accesibilidad Media Global se calcula ajustando la red de infraestructura actual con la propuesta de un túnel que comunica el sector de Parque Centro (sector sur) hasta las cercanías de la actual glorieta de San Rafael (sector norte oriente), con una longitud de aproximadamente $1.9 \mathrm{~km}$. Los resultados permiten identificar que el $25 \%$ del área de estudio se cubre con 27 min., mientras que el $25 \%$ de la población y vivienda se cubre en unos 24 min. de tiempo 
medio de viaje, encontrándose que en relación con el escenario actual, los tiempos medios de viaje disminuyen levemente para un mismo valor de cobertura. En la Fig. 6 se observa la ojiva porcentual de distribución poblacional según estrato y curva isócrona que le cubre.

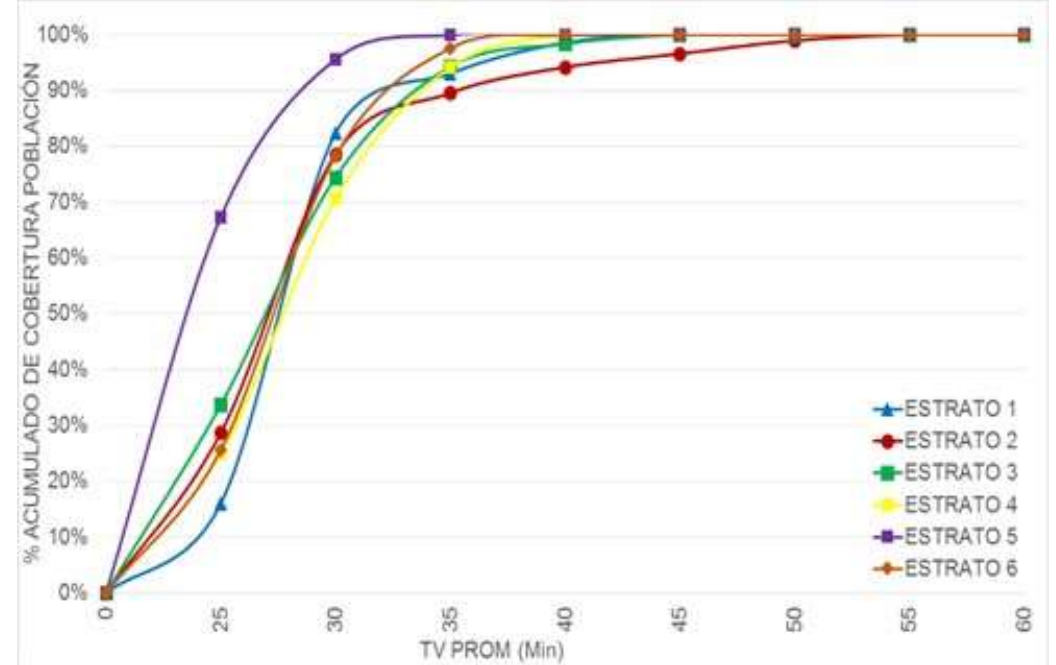

Fig. 6: Distribución porcentual de cobertura de población por estratos para la Alternativa 1

Se destaca nuevamente el estrato 5 como el de mejor cobertura en relación con las características de accesibilidad obtenidas con la implementación de la alternativa 1. Por otra parte, se observa que es el estrato 4 el que reporta condiciones de cobertura más desfavorables. Para una cobertura del $50 \%$ de la población, el estrato 5 se cubriría invirtiendo $23 \mathrm{~min}$. de tiempo medio de viaje, mientras que los estratos 3 , 2, 6, 1 y 4 se cubrirían con tiempos medios de viaje de entre 27 y 28 min., obteniéndose tiempos de viaje prácticamente iguales a los encontrados en el escenario actual para todos los estratos, excepto para el estrato 4.

Al comparar las curvas isócronas del escenario actual con las obtenidas para el escenario de la alternativa 1 , es posible calcular el porcentaje de ahorro de tiempo medio de viaje si se construye dicha alternativa, a lo cual se le denomina gradiente de ahorro de tiempo. En la Fig. 7 se observan los porcentajes de ahorro de tiempo; las zonas en color rojo, son aquellas que no refieren ningún tipo de impacto o ahorro si se construye la alternativa 1, mientras que los sectores en verde son aquellos que tendrían los mayores porcentajes de ahorro, con un valor de hasta un $16 \%$ en relación con el tiempo medio de viaje que se invertiría si no se construyese la obra; este sector está ubicado al sur de la zona de estudio.

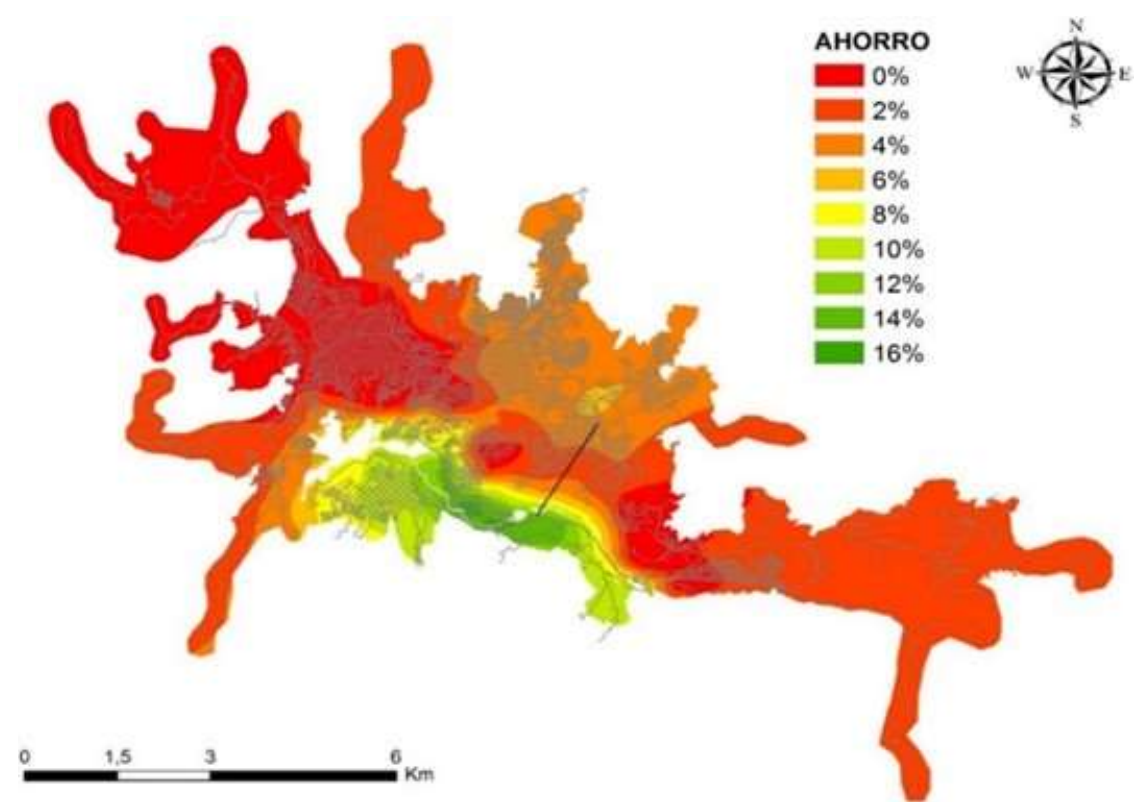

Fig. 7: Gradiente de porcentaje de ahorro para la zona de Estudio, Alternativa 1

En la Fig. 8, se observa la distribución del porcentaje de ahorro en tiempo medio de viaje versus porcentaje de población cubierta con dicho ahorro, valores resultantes del cálculo de gradiente entre escenario actual y alternativa 1. 


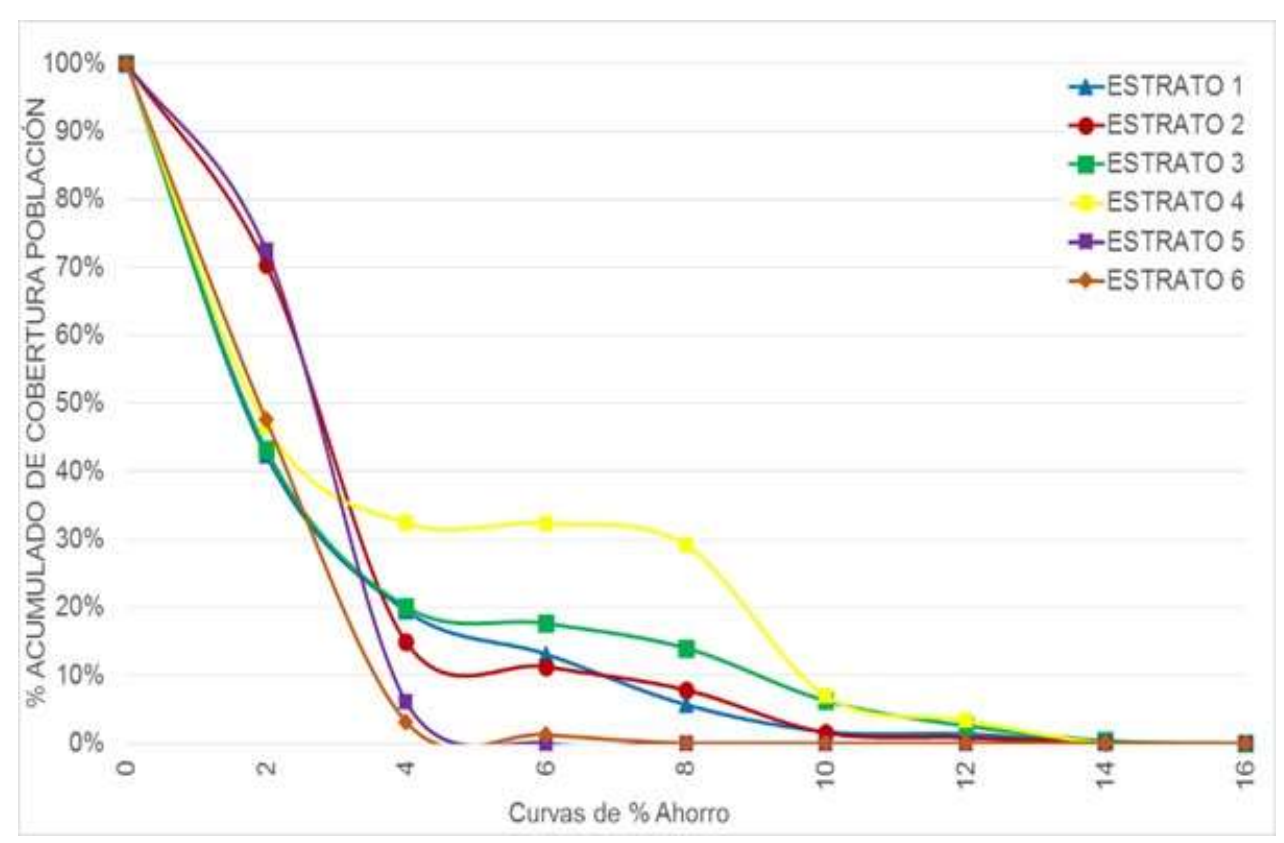

Fig. 8: Porcentaje de ahorro en tiempo Vs porcentaje de población cubierta por estratos para la Alternativa 1

Si se analizan los porcentajes de cobertura poblacional correspondientes a un ahorro del $2 \%$ en el tiempo medio de viaje, se tiene que son los estratos 5 y 2 los más beneficiados, no obstante, al aumentar el porcentaje de ahorro se identifica que es el estrato 4 el que más beneficios tendría si se construyese la alternativa 1, encontrando, por ejemplo, que para un porcentaje de ahorro del $8 \%$ en tiempo medio de viaje, para el estrato 4 se registra una cobertura de hasta un 30\% de población, seguido del estrato 3, 2, 1, 5 y 6 , respectivamente, con porcentajes de ahorro menores al 12\%. En conclusión se tiene que esta alternativa impacta en mayor medida a los estratos 4 y 3 , que refieren porcentajes de cobertura de población con porcentajes de ahorro superiores al 12\%, mientras que los estratos 5 y 6 son sobre los que menor impacto se observa, ya que sus porcentajes de ahorro en tiempo medio de viaje en relación con cobertura poblacional, tan solo llegan hasta un $6 \%$.

Accesibilidad Media Global ofrecida por la Alternativa 2 (Túnel Parque Centro - Barrio Peralonso) y evaluación del impacto en porcentaje de ahorro de tiempo. Como segunda alternativa se propone un túnel desde el sector de Parque Centro (sector sur) hasta las cercanía de la entrada al barrio Peralonso (sector norte), con una longitud aproximada de $3.1 \mathrm{Km}$. Se recalcula el modelo geoestadístico con la inserción de esta nueva infraestructura, obteniéndose los valores de tiempo medio de viaje para la zona de estudio. Los resultados permiten identificar que el $25 \%$ del área de estudio se cubre con 27 min., mientras que el $25 \%$ de la población y vivienda se cubre en unos 24 min. de tiempo medio de viaje, encontrándose que en relación con el escenario actual, los tiempos medios de viaje disminuyen levemente para un mismo valor de cobertura, pero en comparación con los resultados arrojados por la alternativa 1, estos son prácticamente los mismos. Es posible analizar los resultados obtenidos mediante la ojiva porcentual de distribución poblacional según estrato y curva isócrona que le cubre (Ver Fig. 9). De forma general, se observa que es nuevamente el estrato 5 como el de mejor cobertura en relación con las características de accesibilidad obtenidas con la implementación de la alternativa 2, igual resultado obtenido en el escenario actual y en la alternativa 1; no obstante, para esta alternativa, es el estrato 6 el que refiere condiciones de accesibilidad un poco más desfavorables en relación con un $50 \%$ de cobertura de población.

Al comparar las curvas isócronas del escenario actual con las obtenidas para el escenario de la alternativa 2 , es posible calcular el porcentaje de ahorro de tiempo medio de viaje si se construye dicha alternativa, a lo cual se le denomina gradiente de ahorro de tiempo. En la Fig. 10 se observan los porcentajes de ahorro de tiempo obtenidos para este escenario; las zonas en color rojo, son aquellas que no refieren ningún tipo de impacto o ahorro si se construye la alternativa 2, se observa en el sector suroccidente y nororiente de la ciudad, mientras que los sectores en amarillo y verde son aquellos que tendrían los mayores porcentajes de ahorro, con valores que van hasta un $16 \%$ en relación con el tiempo medio de viaje que se invertiría si no se construyese la obra. Si se comparan los resultados obtenidos en relación con los gradientes de ahorro para la alternativa 1 y para la alternativa 2, se identifica claramente que ésta última refiere porcentajes de ahorro de tiempo medio de viaje mayores, mostrando que su impacto en términos de generar una conexión entre los sectores sur y norte de la ciudad, es mayor que el impacto generado por la alternativa 1. 


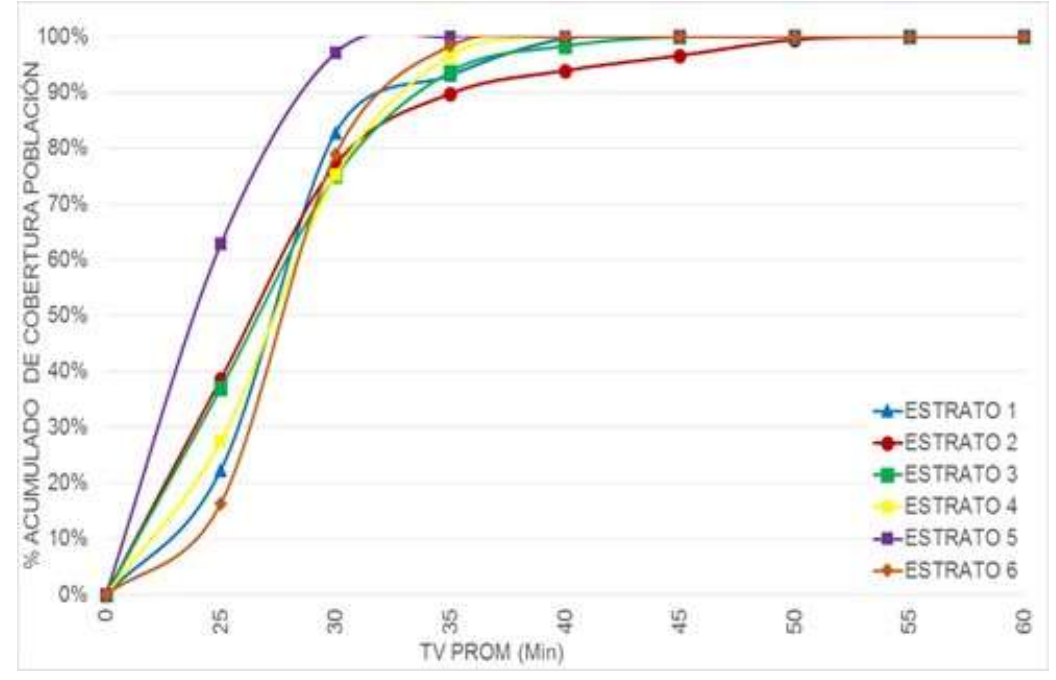

Fig. 9: Distribución porcentual de cobertura de población por estratos para la Alternativa 2

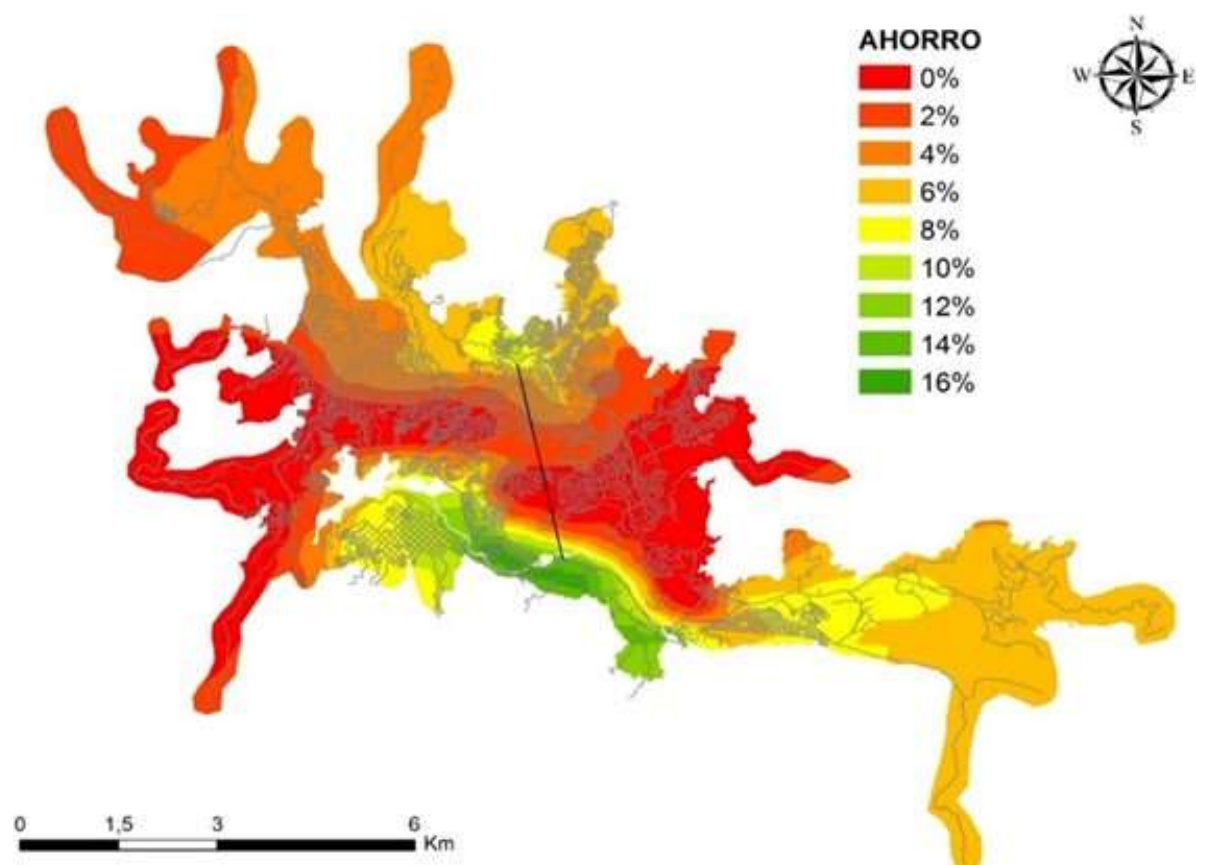

Fig. 10: Gradiente de porcentaje de ahorro para la zona de Estudio

En la Fig. 11, se observa la distribución del porcentaje de ahorro en tiempo medio de viaje versus porcentaje de población cubierta con dicho ahorro, valores resultantes del cálculo de gradiente entre escenario actual y alternativa 2. Si se analizan los porcentajes de cobertura poblacional correspondientes a un ahorro del $2 \%$ en el tiempo medio de viaje, se tiene que son los estratos 4 y 2 los más beneficiados, no obstante, al aumentar el porcentaje de ahorro se identifica que es el estrato 4 el que más beneficios tendría si se construyese la alternativa 2, encontrando, por ejemplo, que para un porcentaje de ahorro del $8 \%$ en tiempo medio de viaje, para el estrato 4 se registra una cobertura superior al $30 \%$ de la población de dicho estrato, seguido del estrato $3,2,1,6$ y 5 , respectivamente, con porcentajes de ahorro menores al $12 \%$.

En conclusión se tiene que esta alternativa impacta en mayor medida a los estratos 4 y 3 , al igual que la alternativa 1, no obstante, en la alternativa 2 se reportan porcentajes de ahorro de tiempo medio de viaje superiores a los obtenidos para la alternativa 1, sólo basta con comparar las curvas para el estrato 4 de la Fig. 8 y la Fig. 11, se identifica cómo la curva reporta un mayor cubrimiento de población para mayores porcentajes de ahorro de tiempo medio de viaje. Así mismo, comparando los demás estratos, se observa, por ejemplo que para un ahorro del $4 \%$ en tiempo medio de viaje los valores de cobertura de población de los estratos 3, 4, 1 y 2 son muy superiores a los obtenidos en la alternativa 1, máxime para el estratos 2 y 1 . 


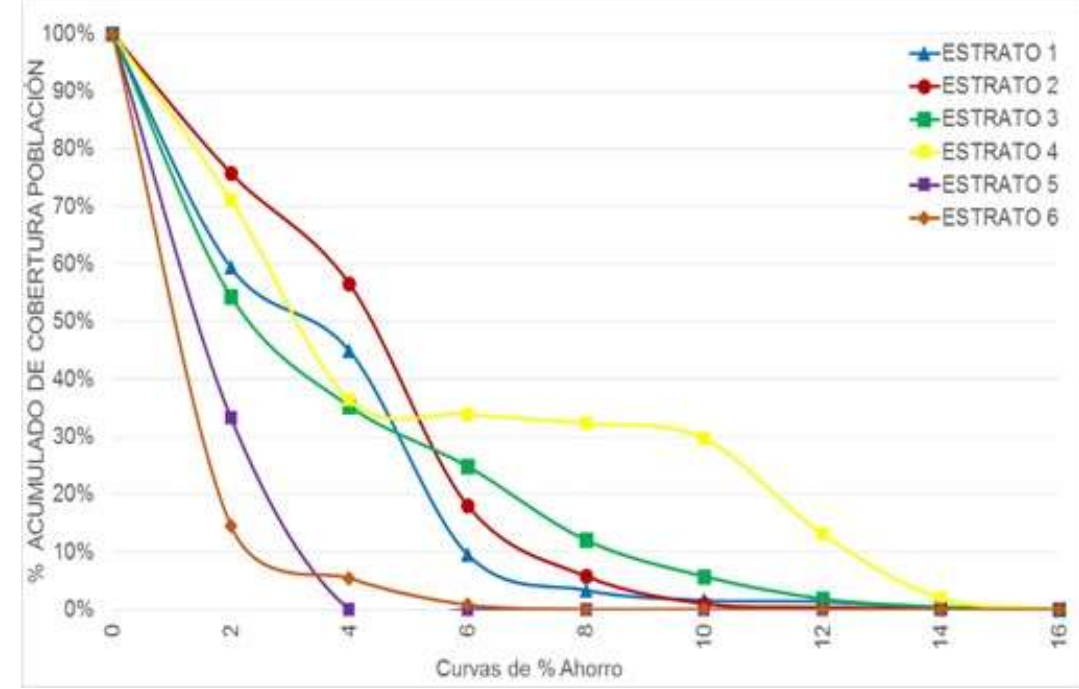

Fig. 11: Porcentaje de ahorro en tiempo Vs porcentaje de población cubierta por estratos para la Alternativa 2

\section{CONCLUSIONES}

A pesar de que se ha comprobado mundialmente que la provisión de nueva infraestructura del transporte tiene pocos efectos sobre la congestión (Duranton y Turner, 2011), ya que no soluciona la misma sino que traslada el sitio donde se presenta, se concluye que al momento de considerar una red de transporte, se debe pensar en cómo las nuevas conexiones ofrecen mejoras en las condiciones de accesibilidad a un territorio o a una parte de éste, lo cual se relaciona directamente en cómo beneficiar a la población más vulnerable y económicamente débil.

Con la alternativa 1 se observa mayor impacto en la zona sur, en el municipio contiguo de Villamaría, mientras que con la alternativa 2, el impacto se observa también en gran parte del sector norte de la ciudad, así como hacia el sector occidente. De forma puntual, en ambas alternativas, los estratos más beneficiados son el 4 y 3 , no obstante, la alternativa 2 reporta mayores impactos que la alternativa 1 . Se tiene que de acuerdo a experiencias de construcción de túneles en Colombia (Caicedo et al., 2009), se calcula un costo de aproximadamente 70 mil millones de pesos por kilómetro, lo cual permite establecer que el costo global de construir la alternativa 1 sería aproximadamente de 133 mil millones de pesos, mientras que construir la alternativa 2 estaría rondando los 217 mil millones de pesos, lo cual es un valor bastante alto para una ciudad que apenas supera los 500 mil millones de pesos de presupuesto anual (Periódico El Espectador, 2015).

Se concluye que los modelos de accesibilidad territorial soportan técnicamente el impacto que sobre los tiempos medios de viaje puede tener una obra de infraestructura como tal y permiten conocer las mejoras que los diferentes sectores de la ciudad tendrían en relación con la inserción de las obras. Es indudable que una propuesta de este tipo mejora las condiciones de accesibilidad y conexión, en este caso en particular, entre los sectores sur y norte de la ciudad, conexión que precisamente es la que actualmente presenta mayores deficiencias. Valdría la pena involucrar modelos de demanda con el fin de conocer cuál es el modo de transporte que se debería fortalecer para hacer uso de esta infraestructura propuesta, teniendo en cuenta que los esfuerzos pueden ser direccionados según las políticas de movilidad expuestas por los gobernantes.

Finalmente, dados los resultados de la evaluación del impacto obtenido por ambas alternativas y articulando esto con los valores de presupuesto referenciados, los autores consideran que la alternativa 1 es sin duda la más factible de ejecutar; no obstante, es necesario resaltar que existen otros modos de transporte que son susceptibles de ser analizados con la presente metodología, por ejemplo, un modo de transporte que seguramente impactaría de una forma positiva las características de movilidad en la ciudad seria el impulsar la construcción de nuevas líneas de cable aéreo, así como complemento decidido de la red de ciclorutas.

\section{AGRADECIMIENTOS}

Los autores expresan su agradecimiento a los estudiantes vinculados a los semilleros de investigación en Movilidad Sostenible y Planificación urbana de la Universidad Nacional de Colombia - Sede Manizales.

\section{REFERENCIAS}

Alcaldía de Manizales, Secretaría de Tránsito y Transporte, Plan de Movilidad de la ciudad de Manizales 2010 - 2040. Universidad Nacional de Colombia - Sede Manizales. Manizales, Colombia (2011) 
Batty, M., Accessibility: in search of a unified theory, doi: 10.1068/b3602ed, Environment and Planning B: Planning and Design, 36,191-194 (2009)

Botero, R., Murillo, J. y Jaramillo, C., Decision strategies for evaluating pedestrian accessibility, https://goo.gl/xRKIhH, Revista Dyna, 78 (168), 28-35 (2011)

Burkey, M., Decomposing geographic accessibility into component parts: methods and an application to hospitals, doi: 10.1007/s00168-010-0415-3, Annals of Regional science, 48 (3), $783-800$ (2012)

Caicedo., J., Balcazar., G y otros veinteseis autores., Colombia avanza en la construcción de túneles, Infraestructura \& Desarrollo, Camara Colombiana de la infraestructura, ISSN 1794-3213, 31, 20-30 (2009)

Chen, S., Claramunt, C. y Ray, C., A spatio-temporal modelling approach for the study of the connectivity and accessibility of the Guangzhou metropolitan network, doi:10.1016/j.jtrangeo.2014.02.006, Journal of Transport Geography, 36,12-23 (2014)

Curl, A., Nelson, J. y Anable, J., Does Accessibility Planning address what matters? doi.org/10.1016/j.rtbm.2012.04.003, Research in Transportation Business \& Management, 3, 84 (2012)

Duranton, G. y Turner, M., Fundamental Law of Road Congestion: Evidence from U.S. Cities, doi:10.1257/aer.101.6.2616, American Economic Review, 101(6), 2616-2652 (2011)

Escobar, D., Cadena, C. y Salas, A., Cobertura Geoespacial de nodos de actividad primaria. Análisis de los aportes a la sotenibilidad urbana mediante un estudio de accesibilidad territorial, doi:10.14508/reia.2015.12.23.13-27, Revista EIA, 12(23), 13-27 (2015a)

Escobar, D., García, F. y Tolosa, R., Diagnóstico de la Movilidad urbana de Manizales, 1aㅡ Edición, Universidad Nacional de Colombia. Facultad de Ingeniería y Arquitectura, Manizales, Colombia (2012)

Escobar, D., García F. y Tolosa R., Análisis de Accesibilidad Territorial a Nivel Regional, 1aㅡ Edición, Universidad Nacional de Colombia. Facultad de Ingeniería y Arquitectura, Manizales, Colombia (2013)

Escobar, D., Tapasco, O. y Giraldo, J., Medición de Desempeño del Sistema de Transporte Cable Aéreo de la Ciudad de Manizales en Colombia, usando Tres Enfoques: Analítico, Simulado y de Accesibilidad Urbana, doi:10.4067/S0718-07642015000600020. Inf. Tecnol. [online]. 26(6), 199-210 (2015b)

Escobar, D., Tamayo, J. y Holguín, J., Propuesta metodológica de evaluación del Transporte Público Colectivo a partir de un análisis de accesibilidad global. Caso de estudio: Manizales y Villamaría - Colombia, Revista Espacios, ISNN 0798-1015, 37(24), 17 (2016)

Gómez, M. y Barredor, J., Sistemas de información geográfica y evaluación multicriterio en la ordenación del

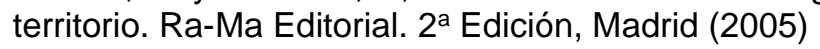

Gutiérrez, J., Condeco-Melhorado, A. y Martín, J., Using Accessibility indicators and GIS to assess spatial spillovers of transport infraestructure investment, doi:10.1016/j.jtrangeo.2008.12.003, Journal of Transport Geography, 18(1), 141-152 (2010)

Halden, D., The use and abuse of accessibility measures in UK passenger transport planning, doi:10.1016/j.rtbm.2011.05.001, Transportation Business \& Management, 2, 12-19 (2011)

Hansen, W., How accessibility shapes land use, doi:10.1080/01944365908978307, Journal of the American Institute of Planners, 25 (2), 73-76 (1959)

Higgs, G., Langford, M. y Fry, R., Investigating variations in the provision of digital services in public libraries using network-based GIS models, doi:10.1016/j.lisr.2012.09.002, Library \& Information Science Research, 35 (1), 24-32 (2013)

Karou, S. y Hull, A., Accessibility modelling: predicting the impact of planned transport infrastructure on accessibility patterns in Edinburgh, UK, doi:10.1016/j.jtrangeo.2014.01.002, Journal of Transport Geography, 35, 1-11 (2014)

Kibambe, J., Radoux, J. y Defourny, P., Multimodal accessibility modeling from coarse transportation networks in Africa, doi.org/10.1080/13658816.2012.735673, International Journal of Geographical Information Science, 27(5) 1005 - 1022 (2013)

Kotavaara, O., Antikainen, H. y Rusanen, J., Population change and accessibility by road and rail networks: GIS and statistical approach to Finland 1970-2007, doi:10.1016/j.jtrangeo.2010.10.013 Journal of Transport Geography, 19 (4), 926-935, (2011) 
Litman, T., Beyond Roadway Level-of-Service: Improving Transport System Impact Evaluation, http://www.vtpi.org/CGOP_LOS.pdf, Victoria Transport Policy Institute (2014)

López, E., Gutierrez, J. y Gómez, G., Measuring regional cohesion effects of large-scale transport infrastructure investment: an accessibility approach, doi: 10.1080/09654310701814629, European Planning Studies, 16 (2), 277-301 (2008)

Mackinnon, D., Pirie, G. y Gather, M., Transport and economic development. In R. Knowles, J. Shaw, \& I. Docherty, Editors, Transport Geographies: Mobilities, Flows and Spaces (10-28). Blackwell Publishers, Oxford (2008)

MCV, Informe de Calidad de Vida Manizales 2015, https://goo.gl/Ees2Ku, Manizales Como Vamos, Manizales (2016)

Moreno, A. y Angulo, J., Desequilibrios y reequilibrios intrametropolitanos: principios de evaluación y metodología de análisis, https://goo.gl/wSxZk6, ISSN: 1133-4762 Ciudad y Territorio Estudios Territoriales, 41(160), 233-262, (2009)

Morris, J., Dumble, P. y Wigan, M., Accessibility indicators in transport planning, https://goo.gl/8UGXYC, Transportation Research, A, 13, 91-109 (1978)

Park, S., Measuring public library accessibility: a case study using GIS, doi:10.1016/j.lisr.2011.07.007, Library \& Information Science Research, 34 (1), 13-21 (2012)

Periódico "El Espectador", Redacción Nacional, Presupuesto de Manizales para 2016 superará los $\$ 500$ mil millones, https://goo.gl/f2HqPI, Acceso: 16 de agosto (2016), Períodico de fecha: 30 Oct. (2015)

Rietveld, P. y Nijkamp P., Transport and regional development. In: J. Polak and A. Heertje, Editors, European Transport Economics, European Conference of Ministers of Transport (ECMT), Blackwell Publishers, Oxford (1993)

Shen, G., Measuring Accessibility of Housing to Public-community Facilities Using Geographical Information Systems, doi: 10.1111/1467-940X.00056, Review of Urban \& Regional Development Studies 14(3), 235-255 (2002)

Vega, A., A multi-modal approach to sustainable accessibility in Galway, doi: 10.1080/20429843.2011.9727923, Regional Insights, 2(2), 15-17, (2011)

Venegas, F. y Rojas, R., Teoría y Práctica del Ordenamiento y Manejo Sustentable del Territorio: TijuanaRosarito-Tecate, Baja California, México, doi:10.1612/inf.tecnol.4077it.08, Inf. Tecnol., 20(3), 73-87 (2009)

Vickerman, R., Spiekermann, K. y Wegener, M., Accessibility and economic development in Europe, doi:10.1080/00343409950118878, Regional Studies, 33 (1), 1-15 (1999)

Vorapracha, P., Phonprasert, P., Khanaruksombat, S. y Pijarn, N., A comparison of Spatial Interpolation Methods for predicting concentrations of Particle Pollution (PM10), https://goo.gl/lo9jo6, International Journal of Chemical, Environmental \& Biological Sciences (IJCEBS), 3(4), 302-306, (2015)

Wong, C. y Webb, B., Planning for infrastructure: challenges to northern England, doi:10.3828/tpr.2014.42, Town Planning Review, 85(6), 683-708 (2014)

Younes, C., Escobar, D. y Holguín, J., Equidad, Accesibilidad y Transporte. Aplicación explicativa mediante un Análisis de Accesibilidad al Sector Universitario de Manizales (Colombia), doi:10.4067/S071807642016000300010. Inf. Tecnol. [online]. 27(3), 107-118 (2016)

Zhu, X. y Liu, S., Analysis of the impact of the MRT system on accesibility in Singapore using an integrated GIS tool, doi:10.1016/j.jtrangeo.2003.10.003, Journal of Transport Geography, 4(12), 89-101 (2004)

Zhang, H. y Gao, Z., Bilevel programming model and solution method for mixed transportation network design problem, doi: 10.1007/s11424-009-9177-3, Journal of Systems Science and Complexity, 22 (3), 446-459, (2009) 